\title{
UM RESULTADO DE PERIODICIDADE PARA UMA EQUAÇÃO INTEGRO-DIFERENCIAL
}

\author{
Rosana Sueli da Motta Jafelice
}

Orientação:

Prof. Dr. Plácido Zoéga Táboas

Dissertação apresentada ao Instituto de Ciências Matemáticas de São Carlos, da Universidade de São Paulo, como parte dos requisitos para obtenção do título de mestre em Matemática.

$$
\text { USP - São Carlos }
$$


Aos meus pais, que acolheram o meu coraçấo, nos seus corações e - geraram para a vida;

Às minhas irmãs, que me ensinaram a partilhar o amor;

Ao Lú, que me incentivou na arte de viver;

E a Deus, pois sem Ele nada disso existiria! 


\section{Agradecimentos}

Ao Prof. Plácido Zoéga Táboas, pela orientação neste trabalho.

À Profa Sandra Maria Semensato de Godoy, pelo apoio técnico e pelas constantes palavras de incentivo.

Aos professores do IBILCE - UNESP, que me mostraram a beleza do mundo matemático.

Ao amigo Castilho que digitou este trabalho como se fosse próprio.

Aos amigos da graduação, da pós-graduação, que tanto me estimularam, e me ajudaram a galgar todos os obstáculos até aqui.

À CAPES pelo apoio financeiro. 


\begin{abstract}
We are concerned with the integro-differential equation:

$$
\dot{x}(t)=-2 \alpha[1+x(t)] \int_{-1}^{-1 / 2} x(t+\theta) d \theta, \quad \alpha>0 . \quad(E)
$$

Our aim is to study the periodic solutions of $(E)$, which are associated to fixed points of a return map $A$ on a closed convex set of the phase

We use a fixed point theorem due to R. Nussbaum to accomplish the existence of nontrivial fixed points of $A$, when $\alpha$ varies along a sequence.
\end{abstract} space. 


\section{Resumo}

Estamos interessados na equação integro-diferencial:

$$
\dot{x}(t)=-2 \alpha[1+x(t)] \int_{-1}^{-1 / 2} x(t+\theta) d \theta, \quad \alpha>0 . \quad(E)
$$

Nosso objetivo é estudar as soluções periódicas de $(E)$, que estão associadas aos pontos fixos de uma aplicação de retorno $A$ sobre um conjunto fechado convexo do espaço de fase.

Nós usamos um Teorema de R. Nussbaum para obter a existência de pontos fixos não triviais de $A$, quando $\alpha$ varia ao longo de uma sequência. 


\section{Conteúdo}

1 Preliminares 3

1.1 Equação Diferencial Funcional com Retardamento . . . . . . . . . . 3

1.2 Existência e Unicidade de Solução para a Equação (0.2) . . . . . . 7

1.3 Um teorema de Periodicidade $\ldots \ldots \ldots \ldots \ldots \ldots \ldots$

2 Existência de uma Solução Periódica não Constante 12

2.1 Fatos Importantes . . . . . . . . . . . . . . . . . 12

2.2 A Equação Característica $\ldots \ldots \ldots \ldots \ldots \ldots \ldots \ldots$

2.3 O Operador de Retorno $\mathrm{A} \ldots \ldots \ldots \ldots \ldots$

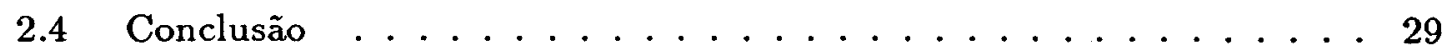




\section{Introdução}

Em 1978, Chow e Hale [1] estudam a equação integro-diferencial

$$
\dot{x}(t)=-\alpha[1+x(t)] \int_{-1}^{0} B(\theta) x(t+\theta) d \theta
$$

onde $\alpha>0$ e $B(\theta) \geq 0$ é de classe $C^{1}$ e semelhante à função delta de Dirac, definida de modo que $B(\theta) \equiv 0, \frac{-1}{2} \leq \theta \leq 0$ e $\int_{-1}^{0} B(\theta) d \theta=1$ (ver Figura 0.1). Esta equação é discutida como um modelo de crescimento de uma espécie. Chow e Hale [1] afirmam que as hipóteses do Teorema 2.2 [2], pag 249, atribuído a R. Nussbaum podem ser verificadas para a equação acima a fim de se obter uma solução periódica não constante.

Inspirados nessa equação e nas afirmações de Chow e Hale, nos detemos em estudar a equação integro-diferencial:

$$
\dot{x}(t)=-2 \alpha[1+x(t)] \int_{-1}^{-1 / 2} x(t+\theta) d \theta, \quad \alpha>0
$$

que é uma equação diferencial funcional com retardamento obtida a partir de (0.1) tomando $B(\theta)$, de acordo com a Figura 0.1 , definida por:

$$
B(\theta)= \begin{cases}2 & \text { se }-1 \leq \theta \leq \frac{-1}{2} \\ 0 & \text { se } \frac{-1}{2}<\theta \leq 0\end{cases}
$$

Por razões técnicas restringimos nosso estudo ao caso em que $\alpha$ pertence a um subconjunto enumerável da reta. 


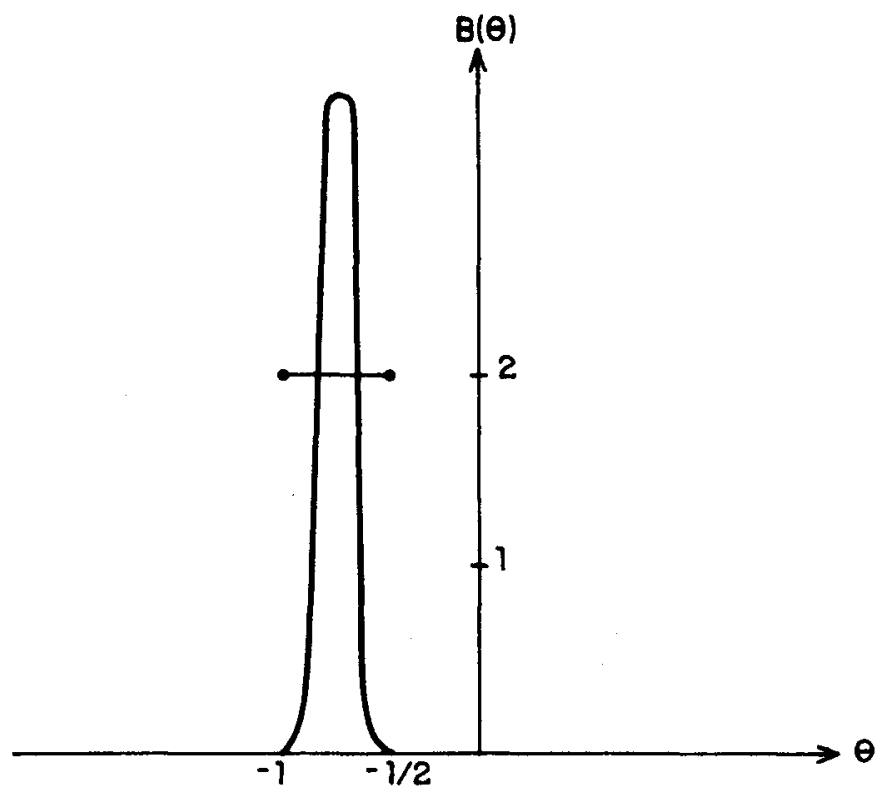

Figura 0.1:

Neste trabalho mostramos a existência de uma solução periódica não constante, usando um princípio de ponto fixo ejetivo para um operador de retorno.

O trabalho é apresentado em dois capítulos. No primeiro citamos alguns resultados básicos para o seu desenvolvimento, definimos equação diferencial funcional com retardamento e apresentamos alguns resultados sobre existência, unicidade e dependência contínua das soluções em relação às condições iniciais. Mostramos a existência de soluções para a equação específica. Definimos ponto ejetivo e enunciamos o Teorema de Nussbaum e um lema que dá uma condição suficiente para um ponto ser ejetivo.

No segundo capítulo, verificamos que a equação específica satisfaz as hipóteses do Teorema de Nussbaum e assim, concluimos a existência de uma solução periódica não constante.

Uma questão que naturalmente se formula a partir do presente trabalho é a de estender o conjunto onde o parâmetro $\alpha$ varia. 


\section{Capítulo 1}

\section{Preliminares}

Apresentamos neste capítulo alguns resultados básicos que serão usados no decorrer do trabalho. As demonstrações podem ser encontradas na bibliografia indicada.

\subsection{Equação Diferencial Funcional com Retardamento}

Consideremos $r \geq 0$ um número real dado, o espaço vetorial n-dimensional $\mathbf{R}^{n}$ sobre os reais com norma euclidiana $|\cdot|, C\left([a, b], \mathbf{R}^{n}\right)$, o espaço de Banach das aplicações $\phi:[a, b] \rightarrow \mathbf{R}^{n}$ contínuas, munido da norma do supremo.

Seja $[a, b]=[-r, 0]$, e $C=C\left([-r, 0], \mathbf{R}^{n}\right)$, onde a norma de um elemento $\phi \in C$ é dada por:

$$
\|\phi\|=\sup _{-r \leq \theta \leq 0}|\phi(\theta)|
$$

Definição 1.1.1 Sejam $\tau \in \mathbf{R}, A \geq 0$ e $x \in C\left([\tau-r, \tau+A], \mathbf{R}^{n}\right)$. Para cada $t \in[\tau, \tau+A]$, definimos a função $x_{t} \in C$ por:

$$
x_{t}(\theta)=x(t+\theta),
$$


onde $-r \leq \theta \leq 0$.

Definição 1.1.2 Sejam $D \subset \mathbf{R} \times C, f: D \rightarrow \mathbf{R}$ uma função, com "."representando a derivada à direita. A equação

$$
\dot{x}(t)=f\left(t, x_{t}\right)
$$

é dita uma equação diferencial funcional com retardamento sobre $D$ e será denotada por EDFR. Se desejarmos enfatizar que a equação é definida por $f$, escrevemos $\operatorname{EDFR}(f)$.

A equação (1.1) inclui como caso particular as equações diferenciais ordinárias, basta para isto tomar $r=0$.

Exemplo 1.1.1 A equação integro-diferencial

$$
\dot{x}(t)=\int_{-r}^{0} g(t, \theta, x(t+\theta)) d \theta
$$

é uma EDFR. Neste caso, $f(t, \phi)=\int_{-\tau}^{0} g(t, \theta, \phi(\theta)) d \theta$ para $(t, \phi) \in \mathbf{R} \times C$.

Definição 1.1.3 Se existe $\tau \in \mathbf{R}$ e $A>0$ tal que $x \in C\left([\tau-r, \tau+A), \mathbf{R}^{n}\right)$, $\left(t, x_{t}\right) \in D$ e $x(t)$ satisfaz a equação (1.1) para $t \in[\tau, \tau+A)$, diz-se que $x$ é solução da equação (1.1).

Definição 1.1.4 Dado $\tau \in \mathbf{R}, \phi \in C$, dizemos que $x(\tau, \phi, f)$ é solução da equação (1.1) com valor inicial $\phi$ em $\tau$ se:

(i) Existe $A>0$ tal que $x(\tau, \phi, f)$ é solução da equação (1.1) sobre $[\tau-r, \tau+A)$,

(ii) $x_{\tau}(\tau, \phi, f)=\phi$. 
Definição 1.1.5 Dizemos que a equação (1.1) é linear se $f(t, \phi)=L(t, \phi)+h(t)$ para $(t, \phi) \in D$, onde $L(t, \phi)$ é linear em $\phi$, para cada $t \in[\tau, \tau+A)$. Dizemos que a equação (1.1) é autônoma se $f(t, \phi)=g(\phi)$, onde $g$ não depende de $t$.

Lema 1.1.1 Se $\tau \in \mathbf{R}, \phi \in C$ são dados e $f(t, \phi)$ é continua, então encontrar uma solução da equação (1.1) passando por $(\tau, \phi)$ é equivalente a resolver a equação integral:

$$
\left\{\begin{aligned}
x_{\tau} & =\phi \\
x(t) & =\phi(0)+\int_{\tau}^{t} f\left(s, x_{s}\right) d s, \quad t \geq \tau .
\end{aligned}\right.
$$

Apresentamos a seguir alguns resultados sobre existência, unicidade e dependência contínua em relação às condições iniciais, das soluções da equação (1.1). As demostrações desses resultados podem ser encontradas em [2].

Se $V \subset \mathbf{R} \times C$, definimos $C^{0}\left(V, \mathbf{R}^{n}\right)$ como sendo o espaço de Banach das funções contínuas e limitadas de $V$ em $\mathbf{R}^{n}$, munido da seguinte norma $|f|_{V}=\sup _{(t, \phi) \in V}|f(t, \phi)|$.

Por inspiração da teoria geral das equações diferenciais ordinárias, a teoria geral dessas equações é desenvolvida em [2], de onde são extraídos os resultados a seguir. Limitamo-nos a apresentar apenas alguns resultados básicos para comodidade do leitor.

Teorema 1.1.1 (Existência) Suponhamos que $\Omega \subset \mathbf{R} \times C$ é aberto e que $f^{0} \in C\left(\Omega, \mathbf{R}^{n}\right)$. Se $(\tau, \phi) \in \Omega$, então existe uma solução da EDFR $\left(f^{0}\right)$ passando por $(\tau, \phi)$. Em geral, se $W \subset \Omega$ é compacto e $f^{0} \in C\left(\Omega, \mathbf{R}^{n}\right)$ são dados, então existe uma vizinhança de $W, V=V(W) \subset \Omega$ tal que $f^{0} \in C^{0}\left(V, \mathbf{R}^{n}\right)$, existe uma vizinhança de $f^{0}, U=U\left(f^{0}\right) \subset C^{0}\left(V, \mathbf{R}^{n}\right)$, e um $\alpha>0$ tal que, para todo $(\tau, \phi) \in W e$ $f \in U$, existe uma solução $x(\tau, \phi, f)$ da EDFR(f) passando por $(\tau, \phi)$ definida em $[\tau-r, \tau+\alpha]$. 
Teorema 1.1.2 (Continuidade em relação às condições iniciais) Suponhamos que $\Omega \subset \mathbf{R} \times C$ é aberto, $\left(\tau^{0}, \phi^{0}\right) \in \Omega, f^{0} \in C\left(\Omega, \mathbf{R}^{n}\right)$ e que a solução $x^{0} d a$ $\operatorname{EDFR}\left(f^{0}\right)$ passando por $\left(\tau^{0}, \phi^{0}\right)$ existe e é única em $\left[\tau^{0}-r, b\right]$. Seja $W^{0} \subset \Omega$ o conjunto compacto, definido por:

$$
W^{0}=\left\{\left(t, x_{t}^{0}\right) \mid t \in\left[\tau^{0}, b\right]\right\}
$$

e seja $V^{0}$ uma vizinhança de $W^{0}$ tal que $f^{0} \in C^{0}\left(V^{0}, \mathbf{R}^{n}\right)$. Se $\left(\tau^{k}, \phi^{k}, f^{k}\right)$ para $k=1,2, \ldots$ satisfaz $\tau^{k} \rightarrow \tau^{0}, \phi^{k} \rightarrow \phi^{0}$ e $\left|f^{k}-f^{0}\right|_{V^{0}} \rightarrow 0$ quando $k \rightarrow \infty$, então existe um $k^{0} \in N$, onde $\operatorname{EDFR}\left(f^{k}\right)$, para $k \geq k^{0}$, é tal que, cada solução $x^{k}=$ $x^{k}\left(\tau^{k}, \phi^{k}, f^{k}\right)$ passando por $\left(\tau^{k}, \phi^{k}\right)$ existe em $\left[\tau^{k}-r, b\right]$ e $x^{k} \rightarrow x^{0}$ uniformemente em $\left[\tau^{0}-r, b\right]$. Como algumas $x^{k}$ podem não estar definidas em $\left[\tau^{0}-r, b\right]$ por $x^{k} \rightarrow x^{0}$ uniformemente em $\left[\tau^{0}-r, b\right]$, entendemos que: para todo $\varepsilon>0, \exists k_{1}=k_{1}(\varepsilon)$ tal que se $k \geq k_{1}, x^{k}(t)$ está definida em $\left[\tau^{0}-r+\varepsilon, b\right]$ e $x^{k} \rightarrow x^{0}$ uniformemente em $\left[\tau^{0}-r+\varepsilon, b\right]$.

Definição 1.1.6 Seja $\Omega \subset \mathbf{R} \times C$ e $f \in C\left(\Omega, \mathbf{R}^{n}\right)$. Dizemos que $f(t, \phi)$ é localmente lipschitziana com relação a $\phi$ em $\Omega$ se $f$ é lipschitziana em $\phi$ para cada subconjunto compacto de $\Omega$, isto é, se $W \subset \Omega$ é compacto, existe $k=k(W)$ de $\mathbf{R}$ tal que para todo $(t, \phi),(t, \psi) \in W$, temos

$$
|f(t, \phi)-f(t, \psi)| \leq k|\phi-\psi|
$$

Teorema 1.1.3 Seja $\Omega \subset \mathbf{R} \times C$ aberto e $f \in C\left(\Omega, \mathbf{R}^{n}\right)$ localmente lipschitziana com relação a $\phi$ em $\Omega$. Se $(\tau, \phi) \in \Omega$, então existe uma única solução da $\operatorname{EDFR}(f)$ passando por $(\tau, \phi)$.

Definição 1.1.7 Suponhamos que $f$ da equação (1.1) é contínua. Se $x$ é uma solução da $\operatorname{EDFR}(f)$ no intervalo $[\tau, a), a>\tau$, dizemos que a função $\hat{x}$ é uma 
continuação ( $a$ direita ) de $x$, se existe $b>a$, tal que $\hat{x}$ está definida em $[\tau-r, b)$, coincide com $x$ em $[\tau-r, a)$ e satisfaz a equação (1.1) em $[\tau, b)$. Dizemos que $x$, solução da equação (1.1) em [ $\tau, a)$, é não continuável (à direita) se não existe uma continuação dela, neste caso o intervalo $[\tau, a)$ é dito intervalo maximal de existência da solução.

O leitor encontra em [2] um estudo bastante geral sobre continuação de soluções.

\subsection{Existência e Unicidade de Solução para a Equação (0.2)}

Esta equação é dada por:

$$
\dot{x}(t)=-2 \alpha[1+x(t)] \int_{-1}^{-1 / 2} x(t+\theta) d \theta, \quad \alpha>0 .
$$

Dado $\phi \in C=C([-1,0], \mathbf{R})$, vamos mostrar que (0.2) admite uma única solução $x(t)$ para $t \geq-1$, tal que $x_{0}=\phi$. Façamos a seguinte mudança de variável:

$$
e^{w(t)}=1+x(t)
$$

$\operatorname{assim} x(t)>-1$ para $t \geq-1$. Além disso,

$$
\dot{x}(t)=e^{w(t)} \cdot \dot{w}(t) .
$$

Por outro lado, de acordo com (0.2),

$$
\dot{x}(t)=-2 \alpha e^{w(t)} \int_{-1}^{-1 / 2}\left[e^{w(t+\theta)}-1\right] d \theta .
$$


Igualando os dois membros, temos que:

$$
\dot{w}(t)=2 \alpha \int_{-1}^{-1 / 2}\left[1-e^{w(t+\theta)}\right] d \theta
$$

Dado $\psi \in C$, verifiquemos que a equação (1.4) admite uma única solução que a satisfaz para $t \geq 0, \operatorname{com} w_{0}=\psi$. Para $t \in[0,1 / 2]$

$$
\dot{w}(t)=2 \alpha \int_{-1}^{-1 / 2}\left[1-e^{\psi(t+\theta)}\right] d \theta
$$

Como o segundo membro é uma função contínua de $t, w(t)$ é determinado por quadratura em $[0,1 / 2]$. Para $t \in[1 / 2,1], w(t)$ é determinado de modo análogo usando $w_{1 / 2}(\theta)=w(1 / 2+\theta),-1 \leq \theta \leq 0$, obtemos $w$ sobre $[-1, \infty)$. Consequentemente, obtemos $x$ sobre $[-1, \infty)$.

\subsection{Um teorema de Periodicidade}

A seguir enunciamos um resultado de Nussbaum, que nos garante a existência de um ponto fixo de uma aplicação $A$ distinto de um ponto prefixado, denominado ejetivo.

Definição 1.3.1 Seja $K$ um conjunto convexo. Um ponto $x_{0} \in K$ é um ponto extremo de $K$ se não existem pontos distintos $x_{1}, x_{2} \in K$ e $t \in(0,1)$ de modo que $x_{0}=t x_{1}+(1-t) x_{2}$. Geometricamente, $x_{0}$ é extremo se ele não é interior de nenhum segmento contido em $K$.

Exemplo 1.3.1 Consideremos $K$ um quadrado. Então os vértices do quadrado serão os pontos extremos de $K$. 
Definição 1.3.2 Seja $X$ um espaço de Banach, $U \subset X$ e $x_{0} \in U$. Dada uma aplicação $A: U-\left\{x_{0}\right\} \rightarrow X$, o ponto $x_{0} \in U$ é dito um ponto ejetivo de $A$ se existe uma vizinhança aberta $G \subseteq X$ de $x_{0}$ tal que para todo $y \in G \cap U, y \neq x_{0}$, existe um inteiro $m=m(y)$ tal que $A^{m} y \notin G \cap U$.

Exemplo 1.3.2 Consideramos $U=X=\mathbf{R}$ e $A: \mathbf{R}-\{0\} \rightarrow \mathbf{R}$ dada por $A(x)=\frac{1}{x}$. Então $x_{0}=0$ é ponto ejetivo de $A$, pois tomando-se $G=G(0)=(-1,1)$ segue que para $y \in G-\{0\}, A y \notin G$, logo basta tomar $m=1$. A vizinhança $G$, neste caso, pode ser qualquer intervalo aberto em $(-1,1)$ que contenha $x_{0}=0$.

Definição 1.3.3 Sejam $X, Y$ espaços de Banach e $f: A \subset X \rightarrow Y$. A função $f$ é completamente continua se for contínua e leva cada limitado de $A$ em um conjunto relativamente compacto de $Y$, isto é, $f$ é compacta.

Seja $K$ um espaço métrico compacto e $M$ um espaço métrico. Considera$\operatorname{mos} C(K, M)$ o conjunto das aplicações contínuas de $K$ em $M$ com topologia dada por:

$$
\mathrm{d}(f, g)=\max _{x \in K} \mathrm{~d}(f(x), g(x))
$$

onde $f, g \in C(K, M)$.

Definição 1.3.4 Um conjunto $E \subset C(K, M)$ é equicontínuo se, para todo $\varepsilon>0$ e para todo $y \in K$, existe $\delta>0, \delta=\delta(\varepsilon, y)$ tal que se $d(x, y)<\delta$, então $d(f(x), f(y))<\varepsilon$, para todo $f \in E$.

Damos a seguir uma versão do Teorema de Ascoli, útil aos nossos propósitos.

Teorema 1.3.1 (Ascoli - Arzelá) Sejam $K$ um espaço métrico compacto e $E \subset C(K, \mathbf{R}) . S e$ 
(i) Existe $N \geq 0$ tal. que $|f(x)| \leq N, \forall x \in K, \forall f \in E$,

(ii) E é equicontínuo,

então E é relativamente compacto.

Para qualquer $M>0$, nós temos $S_{M}=\{x \in X|| x \mid=M\}$ e $B_{M}=\{x \in X|| x \mid<M\}$, então $S_{M}=\partial B_{M}$.

Teorema 1.3.2 (Nussbaum) Seja $X$ um espaço de Banach e $K \subset X$ convexo e fechado. Se $A: K-\{0\} \rightarrow K$ é completamente contínua, $0 \in K$ é um ponto ejetivo de $A$ e existe $M>0$ tal que se $A x=\lambda x$, para $x \in K \cap S_{M}$ implicar $\lambda<1$, então $A$ tem um ponto fixo em $K \cap B_{M}-\{0\}$, se $K$ é de dimensão infinita ou 0 é um ponto extremo de $K$.

Apresentamos a seguir um lema que dá condiçôes para que o 0 seja um ponto ejetivo de $A$.

Consideremos as equações diferenciais retardadas:

$$
\begin{gathered}
\dot{x}(t)=L x_{t}+f\left(x_{t}\right) \\
\dot{y}(t)=L y_{t}
\end{gathered}
$$

onde $L: C \rightarrow \mathbf{R}^{n}$ é uma aplicação linear e contínua, $f: C \rightarrow \mathbf{R}^{n}$ é completamente contínua com a derivada contínua, $f(0)=0$ e $D f(0)=0$. A equação característica da equação (1.6) é dada por:

$$
\operatorname{det} \Delta(\lambda)=0
$$

onde $\Delta(\lambda)=\lambda I-L\left(e^{\lambda} I\right)$. Suas raízes serão chamadas indistintamente raizes características ou autovalores. 
Para cada autovalor $\lambda$, o espaço de fase $C$ é decomposto como soma direta, $C=P_{\lambda} \oplus Q_{\lambda}$, onde $P_{\lambda}$ e $Q_{\lambda}$ são invariantes sob o operador solução da equação (1.6), $T(t), t \geq 0$, dado por $T(t) \phi=y_{t}(\cdot, \phi), \phi \in C$, e $\pi_{\lambda}$ o operador projeção sobre $P_{\lambda}$ definido por essa decomposição.

Lema 1.3.1 Suponhamos que as seguintes condições estejam satisfeitas:

(i) Há um autovalor $\lambda$ da equação (1.6) com $\operatorname{Re}(\lambda)>0$.

(ii) Há um subconjunto convexo fechado $K$ de $C, 0 \in K$, e uma função contínua $\tau: K-\{0\} \rightarrow[\rho, \infty), \rho>0$, tal que a aplicaşão $A: K \rightarrow C$ dada por:

$$
A \phi=\left\{\begin{array}{cl}
x_{\tau(\phi)}(\cdot, \phi), & \phi \in K-\{0\} \\
0, & \phi=0
\end{array}\right.
$$

é completamente contínua e $A K \subset K$.

(iii) $\inf \left\{\left\|\pi_{\lambda} x_{t}\right\| \quad \mid \quad x_{t}=x_{t}(\cdot, \phi), \phi \in K,\|\phi\| \geq \delta, 0 \leq t \leq \tau(\phi)\right\}>0$

(iv) Se $G$ é um subconjunto aberto de $\mathbf{R}^{n}, 0 \in G$, existe uma vizinhança $V$ de 0 em $C$ tal que $x(t, \phi) \in G$, para qualquer $\phi \in V \cap K, \phi \neq 0$, e $0 \leq t \leq \tau(\phi)$.

Então, o é um ponto ejetivo de A. (ver [3]) 


\section{Capítulo 2}

\section{Existência de uma Solução Periódica não Constante}

Utilizando o Teorema 1.3.2 mostramos que (0.2) admite uma solução periódica não constante. Para que esta solução não seja trivial necessitamos de que o ponto fixo de $A$ não seja o zero. Nosso argumento baseia-se em que o zero é um ponto ejetivo de $A$ em $K$, o Lema 1.3 .1 dá as condições necessárias para isso. Primeiro enunciamos alguns fatos importantes, mostramos que as condições do Lema 1.3.1 estão satisfeitas e desta forma, o zero é ponto ejetivo.

\subsection{Fatos Importantes}

O subconjunto fechado e convexo de $C=C([-1,0], \mathbf{R}), K_{\alpha}=\{\phi \in C \mid$ $\phi(-1) \geq 0, \phi(\theta)$ è não decrescente em $[-1,-1 / 2], \phi(\theta)$ é não crescente em $[-1 / 2,0]$, $\left.\phi(0) \geq e^{\frac{-\alpha}{2}\left(e^{\alpha}-1\right)}[\phi(-1 / 2)+1]-1\right\}$ desempenha um papel importante no desenvolvimento do trabalho.

Definição 2.1.1 Dizemos que os zeros de $x(t)$ são limitados se $x(t)$ tem somente um número finito de zeros positivos. 


\section{Lema 2.1.1 .}

Sejam $\phi \in K_{\alpha}$ e $x(t)$ solução da equação (0.2), temos que:

(i ) Se $\phi(0)>-1$ e os zeros de $x(t)$ são limitados, então $x(t) \rightarrow 0$ quando $t \rightarrow \infty$.

(ii) Se $\phi(0)>-1$ e $\alpha>1$, então os zeros de $x(t)$ não são limitados.

(iii) Os zeros de $x(t) \not \equiv 0$ se existem, são simples e a distância de um zero de $x(t)$ ao próximo ponto de máximo, ou de mínimo é $>1 / 2$.

(iv) Se os zeros de $x(t)$ não são limitados então qualquer máximo de $x(t), t>0$ é menor do que $e^{\alpha}-1$.

\section{Prova:}

(i) Suponhamos que existe $t_{1}>0$ tal que $x(t)$ tem sinal constante para $t \geq t_{1}-1$, o que sempre ocorre quando os zeros são limitados. Como $x(t)>-1$ para $t>0$, da seç̧ão 1.2 , temos que:

$$
\dot{x}(t) \int_{-1}^{-1 / 2} x(t+\theta) d \theta=-2 \alpha[1+x(t)]\left[\int_{-1}^{-1 / 2} x(t+\theta) d \theta\right]^{2}<0 \text { para } t \geq t_{1} .
$$

Se $x(t)>0$ para $t \geq t_{1}-1$ então $\dot{x}(t)<0$ para $t \geq t_{1}$, portanto, $x(t)$ é estritamente decrescente e não se anula para $t \geq t_{1}, \operatorname{logo}, x(t) \rightarrow a$, quando $t \rightarrow \infty$, para algum $a \geq 0$.

Se $x(t)<0$ para $t \geq t_{1}-1$ então $\dot{x}(t)>0$ para $t \geq t_{1}$, portanto, $x(t)$ é estritamente crescente e não se anula para $t \geq t_{1}, \operatorname{logo}, x(t) \rightarrow a$, quando $t \rightarrow \infty$, para algum $a \leq 0$.

Desta forma, $x(t)$ é limitada e aproxima-se, monotonicamente de um limite, quando $t \rightarrow \infty$. Suponhamos que $x(t) \rightarrow a$ (>0, para fixarmos um caso) quando 
$t \rightarrow \infty$, então $\dot{x}(t) \rightarrow-\alpha[1+a] a<0$ quando $t \rightarrow \infty$, o que contraria o fato de $x(t) \rightarrow a>0$ quando $t \rightarrow \infty$. Esta contradição leva à conclusão de que $x(t) \rightarrow 0$ quando $t \rightarrow \infty$. O caso $a<0$ é análogo.

(ii) Suponhamos que $\alpha>1$ e os zeros de $x(t)$ são limitados, então por (i) segue que $x(t) \rightarrow 0$ monotonicamente quando $t \rightarrow \infty$, em algum intervalo $\left[t_{1}, \infty\right)$.

Se $x(t)>0$ em $\left[t_{1}, \infty\right)$, temos que:

$$
\begin{aligned}
x\left(t_{1}+1\right)-x\left(t_{1}+1 / 2\right) & =\int_{t_{1}+1 / 2}^{t_{1}+1} \dot{x}(\zeta) d \zeta=\int_{t_{1}+1 / 2}^{t_{1}+1}\left\{-2 \alpha[1+x(\zeta)] \int_{-1}^{-1 / 2} x(\zeta+\theta) d \theta\right\} d \zeta \\
& <-2 \int_{t_{1}+1 / 2}^{t_{1}+1}\left[\int_{-1}^{-1 / 2} x(\zeta+\theta) d \theta\right] d \zeta \leq-\int_{t_{1}+1 / 2}^{t_{1}+1} x(\zeta-1 / 2) d \zeta \\
& <-x\left(t_{1}+1 / 2\right)
\end{aligned}
$$

portanto $x\left(t_{1}+1\right)<0$ ( contradição ). Um raciocínio análogo para $x(t)<0$ também levará a uma contradição. Portanto, os zeros de $x(t)$ não são limitados.

(iii) Seja $z_{0}$ tal que $x\left(z_{0}\right)=0$ e $\bar{\tau}$ o próximo ponto de máximo relativo de $x(t)$, então $\dot{x}(\bar{\tau})=0$. Logo, $\int_{-1}^{-1 / 2} x(\bar{\tau}+\theta) d \theta=0 \Longrightarrow x(\bar{\tau}-1 / 2)>0$. Portanto, $\mathrm{d}\left(z_{0}, \bar{\tau}\right)>1 / 2$. Analogamente, se $\bar{\tau}$ é o próximo ponto de mínimo relativo de $x(t)$.

Mostramos agora que os zeros de $x(t)$ são simples. Seja $\tau_{0}$ um ponto de minimo relativo de $x(t)$ e $z_{0}$ o próximo zero de $x(t)$, então $\dot{x}\left(\tau_{0}\right)=0$. Logo, $\int_{-1}^{-1 / 2} x\left(\tau_{0}+\theta\right) d \theta=0$.

Para $\tau_{0}<t \leq z_{0}$, temos que $\int_{-1}^{-1 / 2} x(t+\theta) d \theta<0$. Portanto, $\dot{x}(t)>0$ para $\tau_{0}<t \leq z_{0}$. Assim, os zeros de $x(t)$ são simples.

O Lema seguinte é usado na demonstração de (iv).

Lema 2.1.2 Se $z_{0}$ é um zero de $x(t), \dot{x}\left(z_{0}\right)>0$ e $\bar{\tau}$ é o próximo ponto de máximo de $x(t)$, então $1 / 2<d\left(z_{0}, \bar{\tau}\right)<1$. 
Prova: Por (iii), temos que : $\mathrm{d}\left(z_{0}, \bar{\tau}\right)>1 / 2$. Supondo que $\mathrm{d}\left(z_{0}, \bar{\tau}\right) \geq 1$. Concluimos que $\dot{x}(\bar{\tau})<0$, uma contradição. Portanto, $1 / 2<\mathrm{d}\left(z_{0}, \bar{\tau}\right)<1$.

(iv) Se $z_{0}$ e $\bar{\tau}$ são como no lema 2.1.2, para $z_{0} \leq t \leq \bar{\tau}$, mostramos que $0 \leq-\int_{-1}^{-1 / 2} x(t+\theta) d \theta<1 / 2$. Por um lado temos que $x(t+\theta)>-1$ para $z_{0} \leq t \leq \bar{\tau}$, logo, $-\int_{-1}^{-1 / 2} x(t+\theta) d \theta<1 / 2$ para $z_{0} \leq t \leq \bar{\tau}$. Por outro lado, $-\int_{-1}^{-1 / 2} x(t+\theta) d \theta \geq 0$, pois $x(t)$ é crescente em $z_{0} \leq t \leq \bar{\tau}$ e $\dot{x}(t) \geq 0$ em $z_{0} \leq t \leq \bar{\tau}$. Portanto:

$$
0 \leq-\int_{-1}^{-1 / 2} x(t+\theta) d \theta<1 / 2, z_{0} \leq t \leq \bar{\tau}
$$

De (2.1) e (0.2), concluimos que $\dot{x}(t)<\alpha[1+x(t)]$. Resolvendo a seguinte equação:

$$
\left\{\begin{array}{l}
\dot{y}(t)=\alpha[1+y(t)] \\
y\left(z_{0}\right)=0
\end{array}, z_{0} \leq t \leq \bar{\tau},\right.
$$

encontramos a solução $y(t)=M e^{\alpha t}-1$, impondo a condição inicial, temos que $M e^{\alpha z_{0}}=1$, ou seja $y(t)=e^{\alpha\left(t-z_{0}\right)}-1$. Sabemos que $y(t)>x(t)$ para $z_{0} \leq t \leq \bar{\tau}$. Logo, $e^{\alpha\left(\bar{\tau}-z_{0}\right)}-1>x(\bar{\tau})$, como $\bar{\tau}-z_{0}<1$, segue que:

$$
x(\bar{\tau})<e^{\alpha}-1 . \square
$$

\subsection{A Equação Característica}

Vamos obter a equação característica da parte linear, em torno de zero, da equação (0.2):

$$
\dot{x}(t)=-2 \alpha \int_{-1}^{-1 / 2} x(t+\theta) d \theta .
$$

Assim, uma condição necessária e suficiente para a existência de uma solução não trivial da equação (2.2), da forma $x(t)=e^{\lambda t} \mu, \mu \in \mathbf{R}-\{0\}$ é que $\mu$ seja uma solução não nula de:

$$
\lambda e^{\lambda t} \mu=-2 \alpha \int_{-1}^{-1 / 2} e^{\lambda(t+\theta)} \mu d \theta
$$


a qual nos leva à equação característica da equação (2.2), dada por:

$$
\lambda^{2}+2 \alpha\left(e^{-\lambda / 2}-e^{-\lambda}\right)=0, \quad \lambda \neq 0
$$

Lema 2.2.1 Se $\alpha$ assume valores ao longo da sequência:

$$
\alpha_{n}=-\frac{a_{n}{ }^{2} e^{a_{n}}}{\operatorname{sen}\left(a_{n}\right)-e^{a_{n} / 2} \operatorname{sen}\left(a_{n} / 2\right)}
$$

onde $\pi+2 n \pi<a_{n}<\frac{4 \pi}{3}+2 n \pi, n=0,1,2, \ldots$ é univocamente determinado, então $\lambda_{n}=a_{n}+i a_{n}$ é uma raiz característica de (2.3).

Prova: Seja equação (2.3) com $\alpha>0$ fazendo $\lambda_{0}=a_{0}+a_{0} i, a_{0} \neq 0$, temos que:

$$
\left(a_{0}+a_{0} i\right)^{2}+2 \alpha\left(e^{\frac{-\left(a_{0}+a_{0} i\right)}{2}}-e^{-\left(a_{0}+a_{0} i\right)}\right)=0 .
$$

Separando a parte real da imaginária, temos que:

$$
\left\{\begin{array}{l}
2 \alpha e^{-a_{0} / 2} \cos \left(\frac{a_{0}}{2}\right)-2 \alpha e^{-a_{0}} \cos \left(a_{0}\right)=0 \\
-2 \alpha e^{-a_{0} / 2} \operatorname{sen}\left(\frac{a_{0}}{2}\right)+2 \alpha e^{-a_{0}} \operatorname{sen}\left(a_{0}\right)=-2 a_{0}^{2}
\end{array}\right.
$$

Multiplicando o sistema por $e^{a_{0}}$, temos que:

$$
\left\{\begin{array}{l}
\cos \left(a_{0}\right)-e^{a_{0} / 2} \cos \left(\frac{a_{0}}{2}\right)=0 \\
\operatorname{sen}\left(a_{0}\right)-e^{a_{0} / 2} \operatorname{sen}\left(\frac{a_{0}}{2}\right)=\frac{-\alpha_{0} e^{a_{0}}}{\alpha}
\end{array}\right.
$$

As equações do sistema (2.4) podem ser escritas como produto escalar:

$$
\begin{array}{r}
\left(\cos \left(a_{0}\right), \cos \left(\frac{a_{0}}{2}\right)\right) \cdot\left(1,-e^{a_{0} / 2}\right)=0 \\
\left(\operatorname{sen}\left(a_{0}\right), \operatorname{sen}\left(\frac{a_{0}}{2}\right)\right) \cdot\left(1,-e^{a_{0} / 2}\right)=\frac{-a_{0}^{2} e^{a_{0}}}{\alpha}
\end{array}
$$

Observe que o vetor $\left(\cos \left(a_{0}\right), \cos \left(a_{o} / 2\right)\right)$ deve ser ortogonal ao vetor $\left(1,-e^{a_{0} / 2}\right)$ e que $a_{0}$ também deve satisfazer a equação (2.6). 
1o Caso : Quando $0<a_{0}<\frac{\pi}{2}$ não satisfaz a equação (2.5), observe:

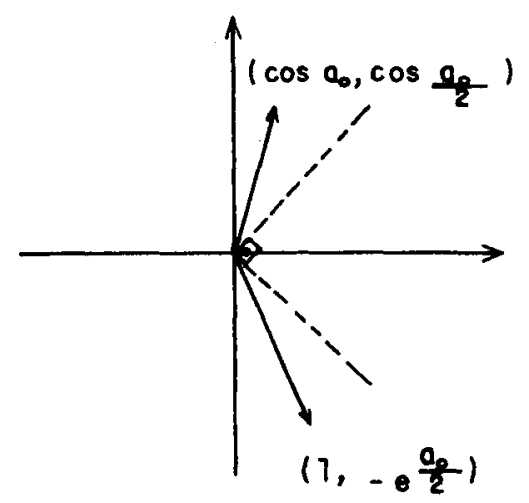

2o Caso : Quando $\frac{\pi}{2}<a_{0}<\pi$ também não satisfaz a equação (2.5), observe:

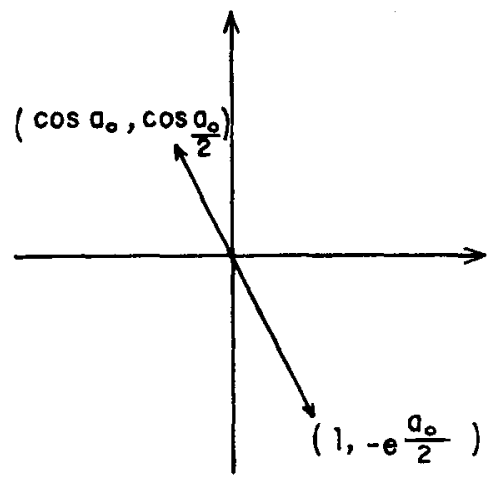

3o Caso : Quando $\pi<a_{0}<\frac{3 \pi}{2}$, concluimos que: existe um único $a_{0}, \pi<a_{0}<\frac{4 \pi}{3}$ que satisfaz a equação (2.5), observe:

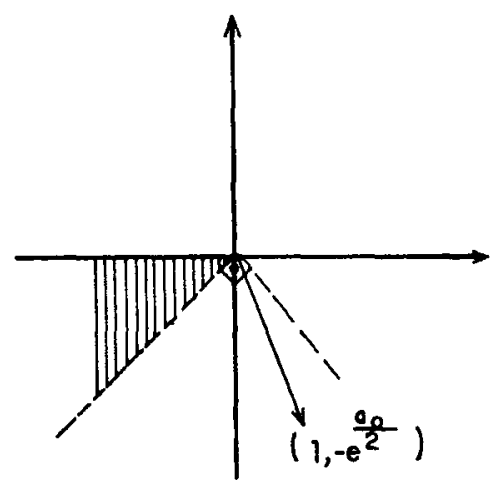

Consequentemente, o vetor $\left(\cos \left(a_{0}\right), \cos \left(a_{0} / 2\right)\right)$ deve estar localizado entre o eixo $x$ e a diagonal do terceiro quadrante, para que seja ortogonal ao vetor 
$\left(1,-e^{a_{0} / 2}\right)$. Portanto, $\pi<a_{0}<\frac{4 \pi}{3}$. Verifiquemos que existe um único $a_{0}$ nessas condições. Para $a_{0}=\pi$, temos que: $\left(\cos \left(a_{0}\right), \cos \left(a_{0} / 2\right)\right) \cdot\left(1,-e^{a_{0} / 2}\right)<0$. Para $a_{0}=\frac{4 \pi}{3}$, temos que: $\left(\cos \left(a_{0}\right), \cos \left(a_{0} / 2\right)\right) \cdot\left(1,-e^{a_{0} / 2}\right)>0$. Além disso, a derivada da função $F\left(a_{0}\right)=\cos \left(a_{0}\right)-e^{a_{0} / 2} \cos \left(a_{0} / 2\right)$ é positiva no intervalo ]$\pi, \frac{4 \pi}{3}\left[\right.$, assim, $F$ é crescente neste intervalo. Portanto, existe um único $a_{0}$, $\pi<a_{0}<\frac{4 \pi}{3}$ que satisfaz a equação $(2.5)$.

4o Caso Quando $\frac{3 \pi}{2}<a_{0}<2 \pi$ não satisfaz a equação (2.5), pois os dois vetores estão no interior do mesmo quadrante.

Para $\pi<a_{0}<\frac{4 \pi}{3}$ satisfaz a equação (2.6), para algum $\alpha=\alpha_{0}$ positivo, dado por:

$$
\alpha_{0}=-\frac{a_{0}^{2} e^{a_{0}}}{\operatorname{sen}\left(a_{0}\right)-e^{a_{0} / 2} \operatorname{sen}\left(a_{0} / 2\right)}
$$

Analogamente concluimos que: $\exists ! a_{n}, \pi+2 n \pi<a_{n}<\frac{4 \pi}{3}+2 n \pi, n=0,1,2, \ldots$, onde:

$$
\alpha_{n}=-\frac{a_{n}^{2} e^{a_{n}}}{\operatorname{sen}\left(a_{n}\right)-e^{a_{n} / 2} \operatorname{sen}\left(a_{n} / 2\right)}
$$

fica univocamente determinado de modo que $\lambda_{n}=a_{n}+i a_{n}$ seja raiz da equação caracteristica (2.3).

Observação 2.2.1 É uma simples, embora extensa, rotina de cálculo, verificar que $\alpha_{n}>1, n=0,1,2, \ldots$

\subsection{O Operador de Retorno A}

Vamos construir um operador de retorno $A: K_{\alpha} \rightarrow C$, onde $K_{\alpha}$ é definido na seç̧ão 2.1,seguindo a órbita a partir de $\phi \in K_{\alpha}$, até o instante em que ela retorna 
a $K_{\alpha}$. Pelo Lema 2.1.1, temos que: se $\phi(0)>-1$ e $\alpha>1$ os zeros de $x(t)$ não são limitados. Com $\alpha_{n}>1, \phi \in K_{\alpha}, \phi \neq 0$, definimos:

$$
\begin{gathered}
\beta(\phi)=\min \{t>0 \mid t \text { é um zero com } \dot{x}(t)>0\} \\
\tau_{1}(\phi)=\{\text { o primeiro ponto de máximo relativo }>\beta(\phi)\} .
\end{gathered}
$$

Sejam $\tau=\tau(\phi)=\tau_{1}(\phi)+1 / 2 \mathrm{e}$

$$
A \phi=\left\{\begin{array}{cl}
x_{\tau(\phi)}(\cdot, \phi), & \phi \in K_{\alpha}-\{0\} \\
0, & \phi=0 .
\end{array}\right.
$$

Lema 2.3.1 A função $\tau: K_{\alpha}-\{0\} \rightarrow[\rho, \infty), \rho>0$, definida acima, é contínua e a aplicação $A: K_{\alpha} \rightarrow C$ dada por:

$$
A \phi=\left\{\begin{array}{cl}
x_{\tau(\phi)}(\cdot, \phi), & \phi \in K_{\alpha}-\{0\} \\
0, & \phi=0
\end{array}\right.
$$

é completamente contínua e $A K_{\alpha} \subset K_{\alpha}$.

Prova: Argumentos típicos de continuidade em relação às condições iniciais levam a continuidade da $\tau$.

Para mostrarmos que $A K_{\alpha} \subset K_{\alpha}$, precisamos primeiramente mostrar que sendo $\bar{\tau}$ um ponto de máximo relativo e $h=x(\bar{\tau})$, então:

$$
x(\bar{\tau}+1 / 2) \geq e^{\frac{-\alpha}{2}\left(e^{\alpha}-1\right)}[x(\bar{\tau})+1]-1
$$

$\operatorname{com} \alpha>0$. Seja

$$
\dot{x}(t)=-2 \alpha[1+x(t)] \int_{-1}^{-1 / 2} x(t+\theta) d \theta
$$

e façamos algumas majorações. Tomando $\bar{\tau}<t \leq \bar{\tau}+1 / 2$, temos que:

$$
\begin{gathered}
\int_{-1}^{-1 / 2} x(t+\theta) d \theta \leq \int_{-1}^{-1 / 2} x(t-1 / 2) d \theta=\frac{1}{2} x(t-1 / 2) \\
x(t-1 / 2) \leq x(\bar{\tau}) \leq e^{\alpha}-1
\end{gathered}
$$


Assim, concluimos que: $\dot{x}(t) \geq-\alpha[1+x(t)]\left(e^{\alpha}-1\right)$. Resolvendo a equação:

$$
\left\{\begin{array}{l}
\dot{y}(t)=-\alpha[1+y(t)]\left(e^{\alpha}-1\right) \\
y(\bar{\tau})=h
\end{array}\right.
$$

Temos que: $\dot{y}(t)+\alpha\left(e^{\alpha}-1\right) y(t)=-\alpha\left(e^{\alpha}-1\right)$. Multiplicando ambos os membros por $e^{\alpha\left(e^{\alpha}-1\right) t}$, temos que:

$$
\left(e^{\alpha\left(e^{\alpha}-1\right) t} y(t)\right)^{\bullet}=-\alpha\left(e^{\alpha}-1\right) e^{\alpha\left(e^{\alpha}-1\right) t} .
$$

Integrando de $\bar{\tau}$ a $t$, obtemos:

$$
e^{\alpha\left(e^{\alpha}-1\right) t} y(t)-e^{\alpha\left(e^{\alpha}-1\right) \bar{\tau}} y(\bar{\tau})=e^{\alpha\left(e^{\alpha}-1\right) \bar{\tau}}-e^{\alpha\left(e^{\alpha}-1\right) t} .
$$

Dividindo por $e^{\alpha\left(e^{\alpha}-1\right) t}$, temos:

$$
y(t)-e^{\alpha\left(e^{\alpha}-1\right)(\bar{\tau}-t)} y(\bar{\tau})=e^{\alpha\left(e^{\alpha}-1\right)(\bar{\tau}-t)}-1 .
$$

Fazendo $t=\bar{\tau}+1 / 2$, concluimos que:

$$
y\left(\bar{\tau}+\frac{1}{2}\right)=e^{\frac{-\alpha}{2}\left(e^{\alpha}-1\right)}[h+1]-1 .
$$

Portanto:

$$
x\left(\bar{\tau}+\frac{1}{2}\right) \geq e^{\frac{-\alpha}{2}\left(e^{\alpha}-1\right)}[x(\bar{\tau})+1]-1 .
$$

Consequentemente, $A K_{\alpha} \subset K_{\alpha}$, pois:

(i) $A \phi(-1)=x(\phi)\left(\tau_{1}-1 / 2\right)>0$

(ii) $A \phi(\theta)=x(\phi)\left(\tau_{1}+1 / 2+\theta\right)$ é não decrescente em $[-1,-1 / 2]$

(iii) $A \phi(\theta)=x(\phi)\left(\tau_{1}+1 / 2+\theta\right)$ é não crescente em $[-1 / 2,0]$

(iv) De (2.7) concluimos que: $A \phi(0)=x(\phi)\left(\tau_{1}+1 / 2\right) \geq e^{\frac{-\alpha}{2}\left(e^{\alpha}-1\right)}\left[x\left(\tau_{1}\right)+1\right]-1$ 
Finalmente, mostremos que $A$ é completamente contínua.

(i) $A \phi$ é contínua, pois, a continuidade de $\tau$ e da solução em relação as condições iniciais garantem a continuidade de $A$ em $K_{\alpha}-\{0\}$. Se $\phi=0 \mathrm{e}$ $\phi_{k} \in K_{\alpha}-\{0\}$, com $\phi_{k} \rightarrow 0$ quando $k \rightarrow \infty$, então, pela continuidade de $\tau$, temos que: $\tau\left(\phi_{k}\right) \rightarrow \tau(0)$ quando $k \rightarrow \infty$. Pela continuidade em relação as condições iniciais para as soluções $x(t)$ da equação $(0.2)$, concluimos que: $x_{\tau\left(\phi_{k}\right)}\left(\phi_{k}\right) \rightarrow x_{\tau(0)}(0)=0$. Portanto, $A \phi$ é contínua em $\phi=0$.

(ii) Mostremos que $A \phi$ é compacta. Seja $\bar{B}_{R}$ uma bola fechada de $C=$ $C([-1,0], \mathbf{R}) \operatorname{com} V=K_{\alpha} \cap \bar{B}_{R}$ e $A V \subset C([-1,0], \mathbf{R})$, onde:

$$
A V=\left\{x_{\tau}(\phi), \phi \in K_{\alpha}-\{0\}, \operatorname{com}\|\phi\| \leq R\right\} \cup\{0\} .
$$

Pelo Teorema 1.3.1 basta mostrar que $A V$ é relativamente compacto, isto é, que $A V$ é limitado e equicontinuo. Pelo Lema 2.1.1, item (iv) temos que: $x(t)<e^{\alpha}-1$ para $t>0$. Seja $L=\max \left(e^{\alpha}-1, R\right)$. $A V$ é limitado, pois $|A \phi(\theta)|=\left|x_{\tau}(\phi)(\theta)\right|=$ $|x(\phi)(\tau+\theta)|<L, \quad \forall \phi \in V$. Para mostrar que $A V$ é equicontínuo, basta provar que: dado $\varepsilon>0, \exists \delta=\delta(\varepsilon)>0$ tal que se $a, b \in[-1,0]$ e $|a-b|<\delta$ então $\left|x_{\tau}(\phi)(a)-x_{\tau}(\phi)(b)\right|<\varepsilon$. Pelo Teorema do Valor Médio, temos que:

$$
\left|x_{\tau}(\phi)(a)-x_{\tau}(\phi)(b)\right| \leq \sup _{-1 \leq \theta \leq-1 / 2}\left|D_{\theta} x(\phi)(\tau(\phi)+\theta)\right||a-b|
$$

Fazendo estimativas para $\left|D_{\theta} x(\phi)(\tau(\phi)+\theta)\right|$ concluimos que :

$$
\sup _{-1 \leq \theta \leq-1 / 2}\left|D_{\theta} x(\phi)(\tau(\phi)+\theta)\right| \leq M, \quad M=\alpha L(1+L), \quad \forall \phi \in V \text { com }\|\phi\| \leq R .
$$

Assim, tomando $\delta=\frac{\varepsilon}{M}$, concluimos que:

$$
\left|x_{\tau}(\phi)(a)-x_{\tau}(\phi)(b)\right|<\varepsilon
$$

isto é, $A V$ é equicontínuo. Pelo Teorema 1.3.1, temos que $A V$ é relativamente compacto. Portanto, $A \phi$ é compacta, logo, $A \phi$ é completamente contínua. 
Vamos verificar, a partir de agora, que o operador $A$ satisfaz à hipótese (iii) do Lema 1.3.1.

Seja $L \phi=-2 \alpha \int_{-1}^{-1 / 2} \phi(\theta) d \theta$ a transformação dada pela linearização (2.3) de $(0.2)$ e $\eta(\theta)$ definida para $\theta \in[-1,0]$ de forma que, sendo

$$
\eta(\theta)=\left\{\begin{array}{cl}
-2 \alpha \theta & -1 \leq \theta \leq-1 / 2 \\
\alpha & -1 / 2<\theta \leq 0
\end{array}\right.
$$

tenhamos:

$$
L \phi=\int_{-1}^{0}[d \eta(\theta)] \phi(\theta),
$$

para todo $\phi \in C$. Seja $G$ o gerador infinitesimal do semigrupo $T(t), t \geq 0$, definido pelas soluções de (2.3), isto é,

$$
T(t) \phi=x_{t}(\cdot, \phi), \phi \in C, t \geq 0
$$

Definição 2.3.1 Dizemos que $\lambda$ é autovalor simples de uma $E D F R(L)$, onde $L$ é linear, se $\lambda$ é uma raiz simples da equação característica da $\operatorname{EDFR}(L)$.

Afirmação 2.3.1 $\lambda_{n}$ é autovalor simples de $G, n=0,1,2, \ldots$.

Prova: Por simplicidade de notação, denotamos $\lambda=\lambda_{n}$ e $\alpha=\alpha_{n}$. Da equação (2.3), temos que:

$$
2 \alpha e^{-\lambda / 2}=-\lambda^{2}+2 \alpha e^{-\lambda}
$$

Seja $f(\lambda)=\lambda^{2}+2 \alpha\left(e^{-\lambda / 2}-e^{-\lambda}\right)$, suponhamos que:

$$
f^{\prime}(\lambda)=2 \lambda-\alpha e^{-\lambda / 2}+2 \alpha e^{-\lambda}=0
$$

Substituindo (2.8) em ( 2.9), concluimos que:

$$
\lambda^{2}+4 \lambda+2 \alpha e^{-\lambda}=0 .
$$


Fazendo $\lambda=a+a i$, temos que:

$$
(a+a i)^{2}+4(a+a i)+2 \alpha e^{-(a+a i)}=0 .
$$

Separando a parte real da imaginária, temos que:

$$
\begin{gathered}
2 a=-\alpha e^{-a} \cos (a) \\
a^{2}+2 a=\alpha e^{-a} \operatorname{sen}(a)
\end{gathered}
$$

Dividindo (2.11) por ( 2.10$)$, temos que:

$$
\frac{a+2}{2}=-\tan (a) \text { para } \pi+2 n \pi<a<\frac{4 \pi}{3}+2 n \pi, n=0,1,2, \ldots \text { ( contradição). }
$$

Portanto, $f^{\prime}(\lambda) \neq 0$, isto é, $\lambda$ é autovalor simples de $G$.

Desta forma, o subespaço $P_{\lambda}$ é precisamente o autoespaço generalizado unidimensional associado a $\lambda, \mathcal{M}_{\lambda}(G)$. Se $G^{*}$ é o operador adjunto formal de $G, \lambda$ é também um autovalor de $G^{*}$ e o autoespaço generalizado $\mathcal{M}_{\lambda}\left(G^{*}\right)$ é também unidimensional. Seja $C^{*}=C([0,1], \mathbf{R})$. A forma bilinear de $C^{*} \times C$, dada por:

$$
\langle\psi, \phi\rangle=\psi(0) \phi(0)-\int_{-1}^{0} \int_{0}^{\theta} \psi(\zeta-\theta)[d \eta(\theta)] \phi(\zeta) d \zeta, \psi \in C^{*}, \phi \in C,
$$

surge de maneira natural na decomposição de $C$, veja ( [2], capítulo 7 ). Seja $\mu$ uma solução não trivial de $\Delta(\lambda) \mu=0$, onde $\Delta(\lambda)$, citado no capítulo 1 , é tal que $\operatorname{det} \Delta(\lambda)=0$ é a equação característica. Logo, $\mu$ é autovetor de (2.2). Tomemos $\mu=1$. Então $\rho(t)=e^{\lambda t}, t \in[-1,0]$, é solução da equação linearizada no espaço $\mathcal{M}_{\lambda}(G)$. Sendo $\nu=\mu=1$ autovetor do operador adjunto $G^{*}$, podemos escrever que $\sigma(s)=e^{-\lambda s}, s \in[0,1]$ é solução da equação linearizada no espaço $\mathcal{M}_{\lambda}\left(G^{*}\right)$. Futuramente, recairemos na decomposição $C=P_{\lambda} \oplus Q_{\lambda}, \forall \phi \in C \operatorname{com} \phi=\phi^{P_{\lambda}}+\phi^{Q_{\lambda}}$, onde a componente $\phi^{P_{\lambda}}$ em $P_{\lambda}$ é dada por $\phi^{P_{\lambda}}=\Pi_{\lambda} \phi=\langle\sigma, \phi\rangle \rho$. Desta forma:

$$
\begin{aligned}
\langle\sigma, \phi\rangle & =\phi(0)-\int_{-1}^{0} \int_{0}^{\theta} e^{-\lambda(\zeta-\theta)}[d \eta(\theta)] \phi(\zeta) d \zeta \\
& =\phi(0)+2 \alpha \int_{-1}^{-1 / 2} \int_{0}^{\theta} \cdot e^{-\lambda(\zeta-\theta)} \phi(\zeta) d \zeta d \theta
\end{aligned}
$$


Já estamos prontos para mostrar que a hipótese (iii) do Lema 1.3 .1 está satisfeita.

Lema 2.3.2 $m=\inf \left\{\left\|\left\langle\sigma, x_{i}\right\rangle\right\| \quad \mid x_{i}=x_{t}(\cdot, \phi), \phi \in K,\|\phi\| \geq \delta, 0 \leq t \leq \tau(\phi)\right\}>0$.

Prova: Vamos supor que $m=0$. Assim, uma sequência $\psi^{n} \in K_{\alpha},\left\|\psi^{n}\right\| \geq \delta$ para $t_{n}, 0 \leq t_{n} \leq \tau\left(\psi^{n}\right), n=1,2, \ldots$, pode ser escolhido de maneira que se $\phi^{n}=x_{t_{n}}=x_{t_{n}}\left(\cdot, \psi^{n}\right)$, então:

$$
\left\|\left\langle\sigma, \phi^{n}\right\rangle\right\|=\left|\phi^{n}(0)+2 \alpha \int_{-1}^{-1 / 2} \int_{0}^{\theta} e^{-\lambda(\zeta-\theta)} \phi^{n}(\zeta) d \zeta d \theta\right| \rightarrow 0, \text { quando } n \rightarrow \infty \text {. }
$$

Nós podemos fazer uma escolha de forma que $\phi^{n}(0) \rightarrow \phi(0)$ quando $n \rightarrow \infty$, e ainda, tomar $\lambda_{0}=a_{0}+a_{0} i \operatorname{com} \pi<a_{0}<4 \pi / 3$, onde:

$$
\alpha_{0}=-\frac{a_{0}^{2} e^{a_{0}}}{\operatorname{sen}\left(a_{0}\right)-e^{a_{0} / 2} \operatorname{sen}\left(a_{0} / 2\right)},
$$

logo,

$$
\begin{aligned}
\left\|\left\langle\sigma, \phi^{n}\right\rangle\right\|= & \mid \phi(0)+2 \alpha_{0} \int_{-1}^{-1 / 2} \int_{0}^{\theta} e^{-a_{0} \zeta+a_{0} \theta} \cos \left(-a_{0} \zeta+a_{0} \theta\right) x\left(t_{n}+\zeta\right) d \zeta d \theta \\
& +i 2 \alpha_{0} \int_{-1}^{-1 / 2} \int_{0}^{\theta} e^{-a_{0} \zeta+a_{0} \theta} \operatorname{sen}\left(-a_{0} \zeta+a_{0} \theta\right) x\left(t_{n}+\zeta\right) d \zeta d \theta \mid .
\end{aligned}
$$

Sejam

$$
\begin{gathered}
\mathcal{R}\left(t_{n}\right)=\phi(0)+2 \alpha_{0} \int_{-1}^{-1 / 2} \int_{0}^{\theta} e^{-a_{0} \zeta+a_{0} \theta} \cos \left(-a_{0} \zeta+a_{0} \theta\right) x\left(t_{n}+\zeta\right) d \zeta d \theta \\
\mathcal{I}\left(t_{n}\right)=2 \alpha_{0} \int_{-1}^{-1 / 2} \int_{0}^{\theta} e^{-a_{0} \zeta+a_{0} \theta} \operatorname{sen}\left(-a_{0} \zeta+a_{0} \theta\right) x\left(t_{n}+\zeta\right) d \zeta d \theta
\end{gathered}
$$

Supondo que $\mathcal{R}\left(t_{n}\right) \rightarrow 0$ quando $n \rightarrow \infty$, vamos mostrar que $\mathcal{I}\left(t_{n}\right)>c$, onde $c>0$. Assim, chegaremos em uma contradição com a hipótese de $m$ ser zero. Além de termos por hipótese $\|\phi\| \geq \delta$, tomemos $\|\phi\| \leq R$ com $R>\delta$. Já provamos que $x(t)<e^{\alpha}-1$ para $t>0$. Desta forma, seja $L=\max \left(e^{\alpha_{0}}-1, R\right)$ e lembremos que $\alpha_{0}>1$. Portanto, $-1<x\left(t_{n}+\zeta\right) \leq L$. 


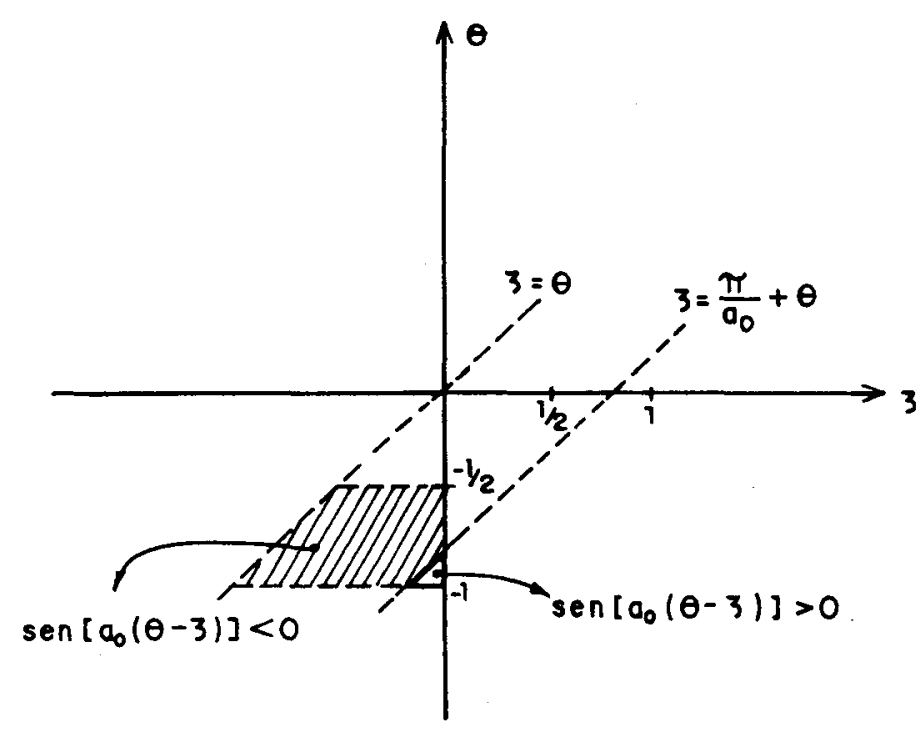

Figura 2.1:

(i) $\operatorname{Para} \operatorname{sen}\left[a_{0}(\theta-\zeta)\right]>0$, temos que:

$$
\begin{gathered}
-2 \alpha_{0} \int_{-1}^{-\pi / a_{0}} \int_{0}^{\pi / a_{0}+\theta} e^{-a_{0} \zeta+a_{0} \theta} \operatorname{sen}\left[a_{0}(\theta-\zeta)\right] d \zeta d \theta< \\
2 \alpha_{0} \int_{-1}^{-\pi / a_{0}} \int_{0}^{\pi / a_{0}+\theta} e^{-a_{0} \zeta+a_{0} \theta} \operatorname{sen}\left[a_{0}(\theta-\zeta)\right] x\left(t_{n}+\zeta\right) d \zeta d \theta \leq \\
2 \alpha_{0} L \int_{-1}^{-\pi / a_{0}} \int_{0}^{\pi / a_{0}+\theta} e^{-a_{0} \zeta+a_{0} \theta} d \zeta d \theta
\end{gathered}
$$

Como podemos ver na figura acima.

(ii) Para $\operatorname{sen}\left[a_{0}(\theta-\zeta)\right]<0$, temos que:

$$
\begin{gathered}
-2 \alpha_{0} \int_{-1}^{-\pi / a_{0}} \int_{\pi / a_{0}+\theta}^{\theta} e^{-a_{0} \zeta+a_{0} \theta} \operatorname{sen}\left[a_{0}(\theta-\zeta)\right] d \zeta d \theta \\
-2 \alpha_{0} \int_{-\pi / a_{0}}^{-1 / 2} \int_{0}^{\theta} e^{-a_{0} \zeta+a_{0} \theta} \operatorname{sen}\left[a_{0}(\theta-\zeta)\right] d \zeta d \theta> \\
2 \alpha_{0} \int_{-1}^{-\pi / a_{0}} \int_{\pi / a_{0}+\theta}^{\theta} e^{-a_{0} \zeta+a_{0} \theta} \operatorname{sen}\left[a_{0}(\theta-\zeta)\right] x\left(t_{n}+\zeta\right) d \zeta d \theta \\
+2 \alpha_{0} \int_{-\pi / a_{0}}^{-1 / 2} \int_{0}^{\theta} e^{-a_{0} \zeta+a_{0} \theta} \operatorname{sen}\left[a_{0}(\theta-\zeta)\right] x\left(t_{n}+\zeta\right) d \zeta d \theta \geq \\
-2 \alpha_{0} L\left[\int_{-1}^{-\pi / a_{0}} \int_{\pi / a_{0}+\theta}^{\theta} e^{-a_{0} \zeta+a_{0} \theta} d \zeta d \theta+\int_{-\pi / a_{0}}^{-1 / 2} \int_{0}^{\theta} e^{-a_{0} \zeta+a_{0} \theta} d \zeta d \theta\right]
\end{gathered}
$$


Assim,

$$
\begin{aligned}
\mathcal{I}\left(t_{n}\right)> & 2 \alpha_{0}\left\{-\int_{-1}^{-\pi / a_{0}} \int_{0}^{\pi / a_{0}+\theta} e^{-a_{0} \zeta+a_{0} \theta} \operatorname{sen}\left[a_{0}(\theta-\zeta)\right] d \zeta d \theta\right. \\
+ & \left.L\left[-\int_{-1}^{-\pi / a_{0}} \int_{\pi / a_{0}+\theta}^{\theta} e^{-a_{0} \zeta+a_{0} \theta} d \zeta d \theta-\int_{-\pi / a_{0}}^{-1 / 2} \int_{0}^{\theta} e^{-a_{0} \zeta+a_{0} \theta} d \zeta d \theta\right]\right\}
\end{aligned}
$$

Seja

$$
\begin{aligned}
f\left(a_{0}\right) & =-\int_{-1}^{-\pi / a_{0}} \int_{0}^{\pi / a_{0}+\theta} e^{-a_{0} \zeta+a_{0} \theta} \operatorname{sen}\left[a_{0}(\theta-\zeta)\right] d \zeta d \theta \\
& =-\frac{e^{-\pi}}{2 a_{0}}\left[\frac{\pi}{a_{0}}-1+\frac{1}{a_{0}}\right]-\frac{e^{-a_{0}} \cos \left(a_{0}\right)}{2 a_{0}^{2}}
\end{aligned}
$$

no gráfico abaixo, observamos que $f(\pi)=0$ e $f\left(a_{0}\right)$ é positiva no intervalo $\left.a_{0} \in\right] \pi, 4 \pi / 3[$.

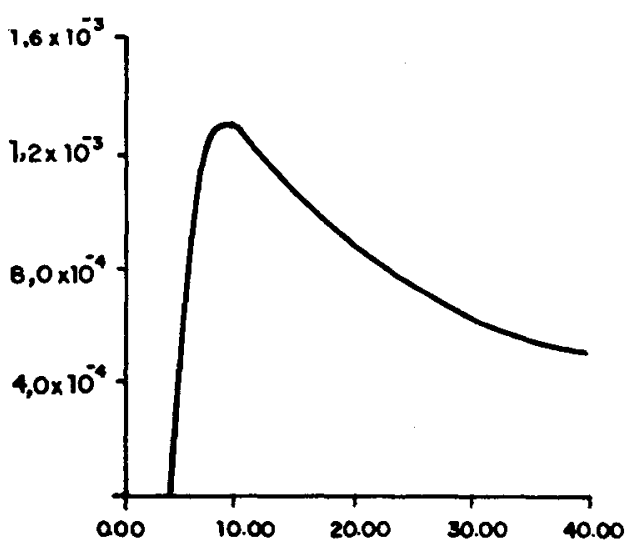

Seja

$$
\begin{aligned}
g\left(a_{0}\right) & =-\int_{-1}^{-\pi / a_{0}} \int_{\pi / a_{0}+\theta}^{\theta} e^{-a_{0} \zeta+a_{0} \theta} d \zeta d \theta-\int_{-\pi / a_{0}}^{-1 / 2} \int_{0}^{\theta} e^{-a_{0} \zeta+a_{0} \theta} d \zeta d \theta \\
& =\left(\frac{-\pi+a_{0}}{e^{\pi} a_{0}^{2}}\right)\left(e^{\pi}-1\right)-\frac{1}{2 a_{0}}+\frac{1}{a_{0}^{2}}\left(\pi-e^{-a_{0} / 2}+e^{-\pi}\right) .
\end{aligned}
$$


No gráfico abaixo, observamos que $g\left(a_{0}\right)>0$ e $g\left(a_{0}\right)$ é decrescente no intervalo $\left.a_{0} \in\right] \pi, 4 \pi / 3[$.

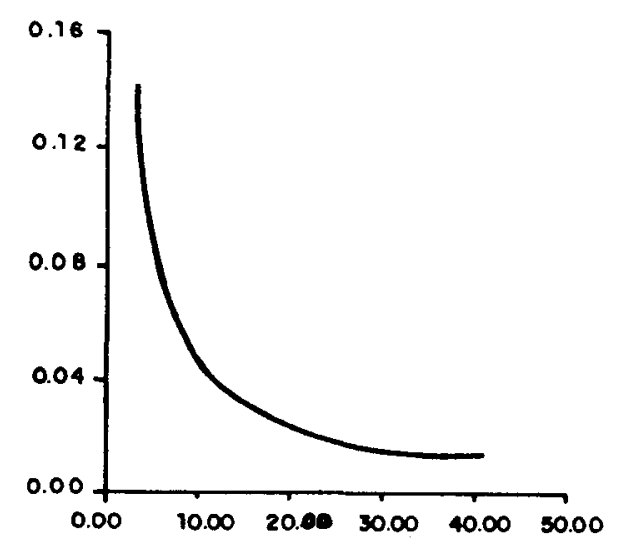

Sendo $f\left(a_{0}\right)+g\left(a_{0}\right)$ é uma função positiva no intervalo $] \pi, 4 \pi / 3[$. Assim, $f\left(a_{0}\right)+L g\left(a_{0}\right)$ também é uma função positiva neste intervalo, porque $L>1$. Portanto, $\mathcal{I}\left(t_{n}\right)>c$, onde $c>0$, no intervalo ] $\pi, 4 \pi / 3[$ (contradição ). Consequentemente, $m>0$.

Assim, analogamente podemos concluir que $m>0$ ao longo da sequência:

$$
\alpha_{n}=-\frac{a_{n}{ }^{2} e^{a_{n}}}{\operatorname{sen}\left(a_{n}\right)-e^{a_{n} / 2} \operatorname{sen}\left(a_{n} / 2\right)}
$$

onde $\pi+2 n \pi<a_{n}<\frac{4 \pi}{3}+2 n \pi, n=0,1,2, \ldots$ univocamente determinado, observando que a área da região onde $\operatorname{sen}\left[a_{n}(\theta-\zeta)\right]$ é positivo tende a zero.

Verifiquemos agora a validade da hipótese (iv) do Lema 1.3.1.

Lema 2.3.3 Se $G$ é um dado subconjunto aberto de $\mathbf{R}, 0 \in G$, existe uma vizinhança $V$ de 0 em $C$ tal que $x(t, \phi) \in G$, para todo $\phi \in V \cap K_{\alpha}, \forall t, 0 \leq t \leq \tau(\phi)$. 
Prova: Seja $\tau_{0}>0$ o primeiro ponto de mínimo relativo de $x(\cdot, \phi)$. Fazendo uma majoração em $(0.2)$, e tomando $0<t \leq \tau_{0}$,

$$
\int_{-1}^{-1 / 2} x(t+\theta) d \theta \leq \int_{-1}^{-1 / 2} \phi\left(\frac{-1}{2}\right) d \theta=\frac{1}{2} \phi\left(\frac{-1}{2}\right) .
$$

Assim, concluimos que $\dot{x}(t) \geq-\alpha[1+x(t)] \phi(-1 / 2)$.

Vamos resolver o P.V.I. ordinário

$$
\left\{\begin{aligned}
\dot{y}(t) & =-\alpha[1+y(t)] \phi(-1 / 2) \\
\phi(-1 / 2) & =H . \\
\dot{y}(t) & +\alpha H y(t)=-\alpha H .
\end{aligned}\right.
$$

Multiplicando ambos os membros por $e^{\alpha H t}$, obtemos:

$$
\left(e^{\alpha H t} y(t)\right)^{\bullet}=-\alpha H e^{\alpha H t} .
$$

Integrando de 0 a $t$,

$$
e^{\alpha H t} y(t)-e^{\alpha H 0} y(0)=e^{\alpha H 0}-e^{\alpha H t} .
$$

Dividindo por $e^{\alpha H t}$ :

$$
y(t)=e^{-\alpha H t} \phi(0)+e^{-\alpha H t}-1 .
$$

Fazendo $t=\tau_{0}$, concluimos que:

$$
y\left(\tau_{0}\right)=e^{-\alpha \phi(-1 / 2) \tau_{0}}[\phi(0)+1]-1 .
$$

Portanto, $0 \geq x\left(\tau_{0}\right) \geq e^{-\alpha \phi(-1 / 2) \tau_{0}}[\phi(0)+1]-1$. Fazendo a mesma majoração anterior em $(0.2)$ e tomando $-1 / 2<t \leq 0$, concluimos que:

$$
\phi(0) \geq e^{\frac{-\alpha \phi(-1 / 2)}{2}}[\phi(-1 / 2)+1]-1 .
$$

Seja $\phi_{n} \in K_{\alpha}$ tal que $\phi_{n}(-1 / 2) \rightarrow 0$ quando $n \rightarrow \infty$. Aplicando o Teorema de Confronto concluimos que $x\left(\phi_{n}\right)\left(\tau_{0}\right) \rightarrow 0$ quando $n \rightarrow \infty$. Tomando $\tau_{1}$ o primeiro 
ponto de máximo relativo, vamos mostrar que se $x\left(\phi_{n}\right)\left(\tau_{0}\right) \rightarrow 0$ quando $n \rightarrow \infty$, então $x\left(\phi_{n}\right)\left(\tau_{1}\right) \rightarrow 0$ quando $n \rightarrow \infty$. Repetindo o processo usado anteriomente, mostramos que $x\left(\tau_{1}\right) \leq e^{\alpha x\left(\tau_{0}\right)\left(z_{0}-\tau_{1}\right)}-1$. Supondo que $x\left(\phi_{n}\right)\left(\tau_{0}\right) \rightarrow 0$ quando $n \rightarrow \infty$ e aplicando o Teorema do Confronto, concluimos que $x\left(\phi_{n}\right)\left(\tau_{1}\right) \rightarrow 0$ quando $n \rightarrow \infty$. Analogamente, obtemos que:

$$
x\left(\tau_{1}+\frac{1}{2}\right) \geq e^{\frac{-\alpha x\left(\tau_{1}\right)}{2}}\left[x\left(\tau_{1}\right)+1\right]-1 .
$$

Usando o fato que $x\left(\phi_{n}\right)\left(\tau_{1}\right) \rightarrow 0$, quando $n \rightarrow \infty$, concluimos que:

$$
x\left(\phi_{n}\right)\left(\tau_{1}+\frac{1}{2}\right) \rightarrow 0 \text { quando } n \rightarrow \infty .
$$

Portanto, tomando $G$ aberto, $G \subset \mathbf{R}, 0 \in G$, existe uma vizinhança de $0 \mathrm{em}$ $C=C([-1,0], \mathbf{R}) \mid x(t, \phi) \in G, \forall \phi \in V \cap K_{\alpha}, 0 \leq t \leq \tau(\phi)$.

\subsection{Conclusão}

A equação (0.2), satisfaz as condições do Lema 1.3.1, portanto, então podemos afirmar que zero é ponto ejetivo de $A$.

Para mostrarmos que todas as hipóteses do Teorema 1.3.2 estão satisfeitas, resta provarmos a seguinte afirmação.

Afirmação 2.4.1 Existe $M>0$ tal que se $A x=\lambda x$, para $x \in K_{\alpha} \cap S_{M}$ implica que $\lambda<1$.

Prova: Suponhamos que para todo $M>0$, existe $x \in K_{\alpha} \cap S_{M}(0)$ tal que $A x=\lambda x$ e $\lambda \geq 1$. Tomando $M>L$, onde $L=\max \left(e^{\alpha}-1, R\right)$, com $\|\phi\| \leq R$, temos que:

$$
|A x|=|\lambda||x| \geq|x|=M \text {. }
$$


Por outro lado, $|A x|<L$ se $x \in K_{\alpha}$, isto é,$|A x|<L<M$. Desta forma $M>|A x| \geq M$ (Absurdo). Portanto, existe $M>0$ tal que $A x=\lambda x$, para todo $x \in K_{\alpha} \cap S_{M}(0)$ implica que $\lambda<1$.

Desta maneira, temos que $A$ tem um ponto fixo em $K_{\alpha} \cap B_{M}-\{0\}$ e portanto a equação (0.2) tem uma solução periódica não constante. 


\section{Bibliografia}

[1] CHOW S. N. ; HALE J. K. - Periodic solutions of autonomous equation. J. Math. Analysis and Applications, v. 66, p. 495-506, 1978.

[2] HALE J. K. - Theory of functional differential equation. New York, Springer, 1977

[3] TÁBOAS P. - Periodic solution of a planar delay equation. Proccedings of the Royal Society of Edinburgh, v. 116A p. 85-101, 1990. 